\title{
TWO CASES OF DOUBLE MELANOMA OF THE UVEA
}

\author{
MICHAEL J. TAPPIN ${ }^{1}$, M. ANDREW PARSONS ${ }^{2}$, KAREN SISLEY ${ }^{3}$, R. C. REES ${ }^{3}$ and \\ IAN G. RENNIE ${ }^{1}$ \\ Sheffield
}

Uveal melanoma is the most common primary intraocular tumour in adults, with an expected incidence of approximately 7 per million of population per year. ${ }^{1}$ The likelihood of more than one uveal melanoma arising in the same patient, although rare, has been reported previously, ${ }^{2,3}$ and both bilateral ${ }^{2}$ and multiple unilateral ${ }^{3}$ uveal melanomas have been described. We report two patients whom we believe both developed two uveal melanomas in the same eye.

\section{Case 1}

\section{CASE REPORTS}

In December 1987 a 57-year-old white man presented to another unit complaining of blurred vision in the right eye. On examination his corrected visual acuities were $6 / 5$ right and $6 / 4$ left. On the right eye there was a pigmented, lobulated lesion arising from the temporal aspect of the iris from approximately 9 o'clock to 10 o'clock. Gonioscopy showed extension of the lesion into the iridocorneal angle. There was a sentinel vessel in the adjacent sclera. A broad iridectomy was performed, the lesion was excised and submitted for histological examination. Macroscopically a black, lobulated tumour was seen that projected from the anterior surface of the iris. Histologically (Fig. 1) the tumour infiltrated and replaced the iris stroma at its base. The neoplasm consisted of amelanotic and lightly pigmented spindle-shaped and polygonal melanocytes, with large ovoid nuclei and occasional nucleoli. Large blood vessels were prominent in the stroma. As excision appeared complete clinically and histologically no further treatment was felt necessary. The

From: ${ }^{1}$ Departments of Ophthalmology and Orthoptics, ${ }^{2}$ Ophthalmic Sciences Unit and ${ }^{3}$ Institute for Cancer Studies, University of Sheffield, Sheffield, UK.

Correspondence to: Mr I. G. Rennie, Department of Ophthalmology and Orthoptics, Royal Hallamshire Hospital, Glossop Road, Sheffield S10 2JF, UK. patient was reviewed annually with no sign of recurrence.

In August 1992 (5 years later) the patient returned with a 2 month history of reduced visual acuity in the same right eye. He was found to have a large tumour arising from the nasal ciliary body and choroid and was referred to this department for further treatment.

On examination his visual acuity was $6 / 60$ right and $6 / 5$ left. A broad iridectomy was present in the right eye extending temporally from the 8 o'clock to 11 o'clock position. Fundoscopy revealed a retinal detachment nasally overlying a large dark mass which extended from the ciliary body to the optic disc. Digital ultrasonography (Cooper Vision) confirmed the presence of a solid lesion which measured $23 \times 21 \times 13 \mathrm{~mm}$. The clinical diagnosis of a melanoma was made. Systemic examination was otherwise normal. In view of the size of the tumour and poor visual prognosis the eye was enucleated. Immediately after enucleation the globe was opened

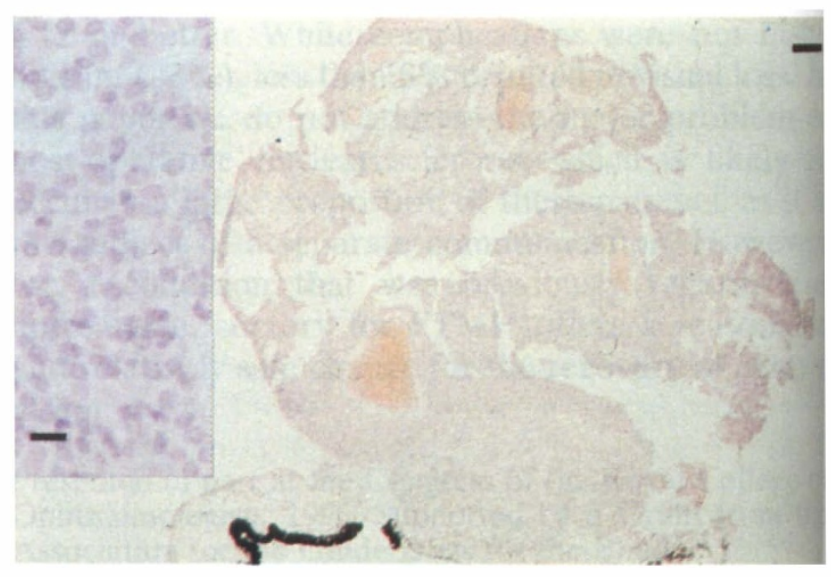

Fig. 1. The iris tumour grows anteriorly from the iris stroma $(H \& E$, scale bar represents $125 \mu \mathrm{m})$. The tumour cells are predominantly amelanotic and are composed of polygonal melanocytes with large ovoid nuclei and occasional nucleoli (insert: $H \& E$, scale bar represents $10 \mathrm{~mm}$ ).

Eye (1996) 10, 600-602 다인 1996 Royal College of Ophthalmologists 


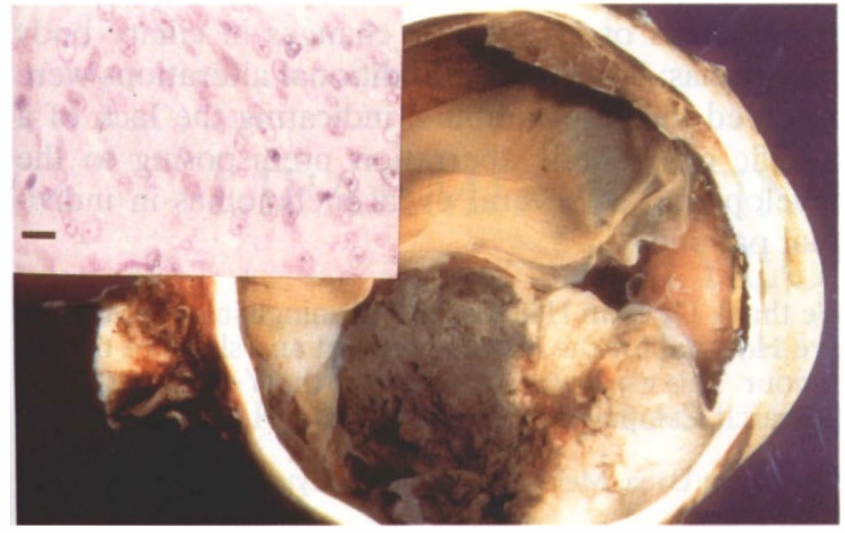

Fig. 2. The nasal ciliary body/choroidal tumour is variegated light grey and black. Tumour cells are spindle $B$ cells, with occasional epithelioid cells (insert: $H \& E$, scale bar represents $10 \mathrm{~mm}$ ).

and a sample was taken from the tumour for cytogenetic analysis as previously described. ${ }^{4}$ Venous blood was also taken for genetic analysis.

Pathological examination showed macroscopically a temporal iridectomy between 8 and 11 o'clock and a dark choroidal tumour nasally, with an overlying retinal detachment. Microscopically (Fig. 2) the anterior surface of the remaining iris showed occasional plaques of atypical melanocytes, the largest of which was situated at the iris root. These cells were similar to the original iris melanoma, and probably represented residual tumour. In contrast the ciliary body/choroidal tumour possessed both spindle B $(90 \%)$ and epithelioid $(10 \%)$ melanocytes, confirming a diagnosis of a melanoma of mixed cell type.

\section{Case 2}

A 64-year-old woman was referred from another centre with a 6 month history of reduced vision in the right eye. On examination her corrected vision was hand movements right and $6 / 5$ left. In the anterior

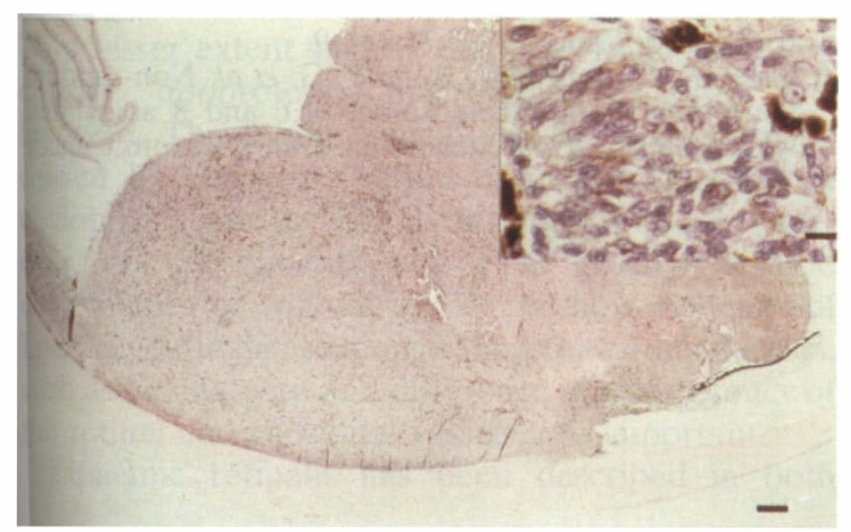

Fig. 3. The inferiorly situated melanoma arises in the ciliary body and peripheral choroid, displaces the iris anteriorly, and infiltrates the iris and anterior chamber drainage angle (H\&E, scale bar represents $0.5 \mathrm{~mm})$. Inset: spindle $B$ melanoma cells (H\&E, scale bar represents $10 \mathrm{~mm})$. chamber of the right eye there was a dense cataract and a dark mass in the iridocorneal angle between 6 o'clock and 9 o'clock. Sentinel vessels could be seen on the adjacent quadrant of sclera. There was no view of the fundus. Digital ultrasonography (Cooper Vision) revealed a mass in the inferotemporal region. This measured $14 \mathrm{~mm}$ across its base and about $14 \mathrm{~mm}$ from base to apex, and an A-scan showed relatively low internal reflectivity. The diagnosis of ciliary body melanoma was made. Clinical investigation showed no evidence of metastases. The eye was enucleated and the globe opened; tissue was sent for cytogenetic analysis as well as histological investigation.

Pathological examination of the globe showed macroscopically a black tumour, $15 \mathrm{~mm}$ anteroposteriorly, replacing the ciliary body and peripheral choroid, displacing the iris and lens anteriorly, and infiltrating the drainage angle. In the peripheral choroid nasally there was a flat black lesion, $1 \mathrm{~mm}$ from the ora serrata and $14 \mathrm{~mm}$ from the closest edge of the main tumour.

On microscopy the main tumour was a lightly pigmented ciliary body and choroidal melanoma of spindle B $(>95 \%)$ cell type, which had invaded the peripheral half of the iris and the anterior chamber drainage angle (Fig. 3). The nasal lesion was a choroidal melanoma composed of cells with moderately pleomorphic ovoid nuclei, some with nucleoli (spindle A and B cells) (Fig. 4).

\section{Cytogenetic Analysis of Cases 1 and 2}

Analysis of blood samples from the patients showed no constitutional cytogenetic alterations which would predispose to uveal melanomas. Chromosome analysis of the choroidal neoplasms revealed the following clonal karyotypes: case $1,44, \mathrm{X},-\mathrm{Y}$, $\operatorname{der}(1) \mathrm{t}(1 ; 8)(\mathrm{q} 10 ; \mathrm{q} 10),-3$; case 2 (the larger of the

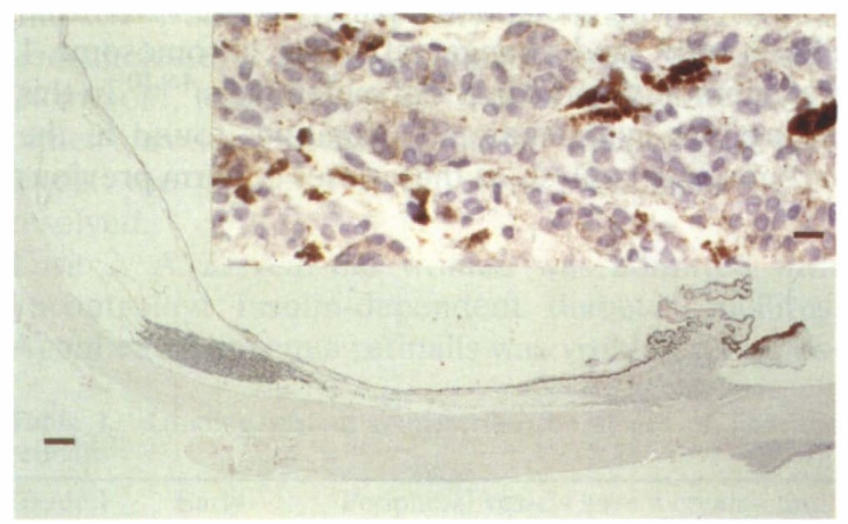

Fig. 4. The small nasal melanoma is confined to the choroid peripherally (H\&E, scale bar represents $0.5 \mathrm{~mm}$ ), and is composed of cells with moderately pleomorphic ovoid and spindle-shaped nuclei, some with nucleoli (inset: $H \& E$, scale bar represents $10 \mathrm{~mm}$ ). 
two tumours), 44,-46,X, $-\mathrm{X}[14], \operatorname{add}(1)(\mathrm{p} 31)[5]$, $-3[14],+8[14], \operatorname{add}(16)(\mathrm{q} 21)[17],+\operatorname{mar} 1[5]+\operatorname{mar} 2[4]$ [cp14]. For both tumours complete loss of one copy of chromosome 3 , or monosomy, was observed in association with an entire extra or partial copy of chromosome 8 . In addition both tumours effectively demonstrated partial deletions for the short arm of chromosome 1 .

\section{COMMENT}

Uveal melanomas are rare. ${ }^{1}$ The likelihood of developing two primary uveal melanomas is approximately 1 in 50 million. ${ }^{2}$ However, we believe both these cases represent a rare finding of two separate tumours affecting the same eye. There was no sign at enucleation of any continuity between the separate tumours and in case 1 they were of markedly different histological type.

Although multiple uveal melanomas are rare there are reports of both unilateral ${ }^{3}$ and bilateral cases. ${ }^{2}$ There have also been a small number of familial cases of uveal melanoma, including a family with three successive generations affected. ${ }^{5}$ These multiple and familial tumours suggest there may be a predisposing genotypic abnormality. There are several conditions known to be associated with an increased incidence of uveal melanoma, including neurofibromatosis, ${ }^{6}$ ocular or oculodermal melanocytosis $^{7}$ and familial cutaneous melanoma. ${ }^{8}$ Clinically, there was no evidence of any of these conditions in these patients. The analysis of the patients' blood did not identify any constitutional cytogenetic alterations which might account for a predisposition to uveal melanoma. Cytogenetic analysis of uveal melanomas has revealed consistent abnormalities of chromosomes 3, 6 and 8. Aberrations affecting chromosomes 3 and 8 , such as the loss of one copy of chromosome 3 with additional copies of the long arm of chromosome 8, are particularly associated with melanomas partly derived from the ciliary body, and changes affecting chromosome 1 are commonly found in uveal melanomas. ${ }^{4,9,10}$ In this respect the cytogenetic abnormalities found in the chromosome analysis of these cases confirm previous observations of cytogenetic changes in ciliary body melanomas; however, no additional alterations were observed in these tumours, indicating the lack of a specific cytogenetic aberration predisposing to the development of several uveal melanomas in individual patients.

We thank Dr John Harry of the Birmingham and Midland Eye Hospital for the histology report and slides of the iris tumour. This work was supported by the Yorkshire Cancer Research Campaign (YCRC).

Key words: Uvea, Melanoma, Tumour, Cytogenetics.

\section{REFERENCES}

1. Wilkes SR, Robertson DM, Kurland LT, Cambell JR. Incidence of uveal melanoma in the resident population of Rochester and Olmstead County, Minnesota. Am J Ophthalmol 1979;87:639-41.

2. Shammas HF, Watzke RC. Bilateral choroidal melanomas: case report and incidence. Arch Ophthalmol 1977;95:617-23.

3. Volcker HE, Naumann GOH. Multicentric primary malignant melanomas of the choroid: two separate malignant melanomas of the choroid, and two uveal naevi in one eye. Br J Ophthalmol 1978;62:408-13.

4. Sisley K, Rennie IG, Cottam DW, et al. Cytogenetic findings on six posterior uveal melanomas: involvement of chromosomes 3, 6 and 8. Genes Chrom Cancer 1990;2:205-9.

5. Walker JP, Weiter JJ, Albert DM, et al. Uveal melanoma in three generations of the same family. Am J Ophthalmol 1979;88:723-6.

6. Wiznia RA, Freedman JK, Mancini AD, Shields J. Malignant melanoma of the choroid in neurofibromatosis. Am J Ophthalmol 1978;89:684-7.

7. Sabates FN, Yamashita T. Congenital melanosis oculi complicated by two independent malignant melanomas. Arch Ophthalmol 1967;77:801-3.

8. Abrahamson DH, Rodriguez-Sains RS, Ruberman R. B-K mole syndrome cutaneous and ocular malignant melanoma. Arch Ophthalmol 1980;80:1397-9.

9. Horsman DE, White V. Cytogenetic analysis of uveal melanoma: consistent occurrence of monosomy 3 and trisomy 8q. Cancer 1993;71:811-9.

10. Sisley K, Cottam DW, Rennie IG, et al. Non-random abnormalities of chromosomes 3, 6 and 8 associated with posterior uveal melanoma. Genes Chrom Cancer 1992;5:197-200. 